\title{
El proceso de internacionalización de la educación superior en Argentina (1995-2015): ¿determinantes exógenos 0 endógenos?
}

MerCedes BotTo*

Artículo recibido: 3 de diciembre de 2016

Artículo aprobado: 19 de julio de 2017

Doi: http://dx.doi.org/10.12804/revistas.urosario.edu.co/desafios/a.5281

Para citar este artículo: Botto, M. (2018). El proceso de internacionalización de la educación superior en Argentina (1995-2015): ¿determinantes exógenos o endógenos? Desafios, 30(1), 215-243. Doi: http://dx.doi.org/10.12804/revistas.urosario.edu.co/desafios/a.5281

\section{Resumen}

El propósito de este artículo es analizar el proceso de internacionalización de la educación superior en Argentina, en el marco del debate sobre los alcances de las reformas estructurales en América Latina. A través de la reconstrucción histórica de la trayectoria seguida por el sistema de educación superior argentino entre 1995 y 2015, se busca contribuir al debate sobre la naturaleza de la reforma y los factores que determinaron sus orígenes y sus alcances. La selección del caso argentino se bace en virtud de sus peculiaridades nacionales, que lo convertirían en un ejemplo de las

\footnotetext{
* Socióloga de la Universidad de Buenos Aires (UBA). Docente de la UBA y la Facultad Latinoamericana de Ciencias Sociales (Flacso). Investigadora independiente del Consejo Nacional de Investigaciones Científicas de la Argentina (CONICET). Correo electrónico: mbotto@flacso.org.ar ORCID: http://orcid.org/0000-0003-4368-2374

La autora quiere agradecer a Agustina Frisch por su asistencia en la edición de este artículo y a los dos revisores que aportaron sus valiosos comentarios.
} 
posibilidades de la reforma en materia de privatización, descentralización y (des) regulación gubernamental. Las conclusiones desmitifican tanto las explicaciones que bablan de una sobredeterminación exógena como de las tesis voluntaristas que bablan de las orientaciones ideológicas y los cálculos políticos de los líderes de gobierno. Palabras clave: educación superior, reformas estructurales, América Latina.

\title{
The Internationalization Process of Higher Education in Argentina (1995-2015): Endogenous or Exogenous Explanations?
}

\begin{abstract}
The purpose of this article is to analyze the process of internationalization of higher education in Argentina, centered around the debate on the scope of the structural reforms in Latin America. Through the historical reconstruction of the path followed by the Argentinean higher education system from 1995 to 2015, we seek to contribute to the debate on the nature of the reform and the factors that determined its origins and scope. The selection of the Argentinean case is due to its national peculiarities, which make it an exemplary case of structural reforms in the areas of privatization, decentralization, and government (de)regulation. The conclusions demystify both the explanations that emphasize an exogenous over-determination as well as the arguments that underline the ideological orientation and political calculation of government leaders.
\end{abstract} Keywords: Higher education, structural reforms, Latin America.

\section{O processo de internacionalização da educação superior na Argentina (1995-2015): determinantes exógenos ou endógenos?}

\begin{abstract}
Resumo
Opropósito do artigo é analisar o processo de internacionalização da educação superior na Argentina, enquadrado no debate sobre os alcances das reformas estruturais na América Latina. Através da reconstrução histórica da trajetória seguida pelo sistema de educação superior argentino entre 1995 e 2015 busca-se contribuir ao debate
\end{abstract}


sobre a natureza da reforma e os fatores que determinaram seus origens e alcances. A eleição do caso argentino se faz em virtude de suas peculiaridades nacionais que o converteriam em um caso exemplificador das possibilidades da reforma em matéria de privatização, descentralização e regulação governamental. As conclusões desmitificam tanto as explicações que falam de uma sobre determinação exógena quanto das teses voluntaristas que falam das orientações ideológicas e os cálculos políticos dos lideres do governo.

Palavras-chave: educação superior, reformas estruturais, América Latina.

\section{Introducción}

La literatura que analiza los procesos de reforma educativa en América Latina tiende a mostrar visiones muy diferentes e incluso contrapuestas sobre los alcances y los resultados de los procesos de internacionalización/transnacionalización iniciados en los años noventa, los cuales se extienden hasta nuestros días. Para una vasta parte de la literatura centrada en los procesos de reforma de los años noventa, se trata de un fenómeno determinado por ideas e instituciones exógenas que, lideradas por el Consenso de Washington y el Banco Mundial (BM), desembarcan en la región y obligan a los distintos Gobiernos a poner en marcha un mismo repertorio de políticas para todo el desarrollo. Aun cuando esta reforma no contó con el beneplácito de los actores domésticos, su puesta en marcha — sin mayores modificacionesestuvo asegurada por la situación de crisis institucional y financiera en que se encontraba el sector educativo (Marquis, 1998; Chiroleu, 2003; Didou Aupetit, 2005; Mollis, 2007).

Para otros autores, en cambio, la implementación exitosa de la reforma estructural es el resultado de la decisión y convencimiento de las elites gubernamentales sobre la necesidad de la misma. En este sentido, lejos de considerarla como una imposición, los líderes del poder ejecutivo y/o de las instituciones de educación superior (IES) abrazan la reforma por considerarla una oportunidad para potenciar su competencia en el marco de la internacionalización y transnacionalización de la educación superior (ES) (de Wit, 2011; Albatch \& Knight, 2006; Tobón, Gallego \& Yepes, 2013). 
Más allá de las diferencias aparentes ${ }^{1}$, en este trabajo consideramos que ambas visiones son la cara y el reverso de una misma moneda que caracteriza la reforma como un hecho cumplido y exitoso antes de su puesta en marcha, que desconocen las restricciones políticas e institucionales que modifican —o pueden hacer peligrar - su puesta en marcha en el ámbito nacional. Visiones semejantes terminan por caracterizar a las reformas como procesos convergentes y a minimizar las diversidades nacionales que se platean en términos de los alcances, las modalidades y los tiempos de las reformas.

Con el fin de ilustrar la importancia de los procesos políticos que acompañan a los procesos de reforma sectorial, proponemos analizar el caso de la reforma de la ES en Argentina entre mediados de los años noventa hasta el 2015, a partir de la manera en que las elites gubernamentales de los distintos gobiernos de turno han mediado entre las presiones externas y las demandas internas, para darle forma y especificidad nacional a los repertorios comunes de reforma elaborados y diseminados desde los distintos foros internacionales. En ese sentido, este análisis se inscribe en la nueva literatura sobre el ajuste estructural en los países en desarrollo, que entiende las opciones que hacen los Gobiernos frente a las coyunturas económicas adversas no como datos exógenos, sino que buscan profundizar sobre la variable endógena, explicando por qué entre las distintas opciones se escogen algunas, y por qué se llega a resultados tan diversos (Kauffman \& Haggard, 1992; Nelson, 1994; Torre, 1998; entre otros).

En este artículo nos proponemos contribuir a este debate, ampliando el análisis de las políticas no económicas, que como la educación y la salud, también formaron parte del paquete de reformas de ajuste sectorial propuestos por los bancos de desarrollo en los años noventa. El interés por focalizar el caso de la política de ES en Argentina por sobre el resto de los países del Cono Sur reside en la importancia de

\footnotetext{
1 La primera considera que no hay otra opción posible: la reforma viene determinada desde afuera y por lo tanto su resultado exitoso es previsible y necesario. Para la segunda, la reforma es solo un acto administrativo y en ella solo importa la voluntad de los ejecutantes con el libreto prefijado. En este caso, el compromiso de la dirección (del ejecutivo nacional o de las IES) es la llave para el éxito de la reforma.
} 
la universidad pública como actor relevante en la historia política y social del país. Este poder estaba apoyado sobre tres principios: el de la inclusión (ingreso irrestricto), el de la autonomía académica y el del financiamiento público. Sin embargo, a partir de los años sesenta, este modelo empezó a desbarrancarse por las sucesivas intervenciones de gobiernos militares y por la falta de financiamiento para dar respuesta a una demanda creciente de la matrícula. En los años noventa, se inicia un proceso de reforma estructural que, siguiendo un repertorio común de reformas en toda América Latina, logró trastocar las bases de la autonomía universitaria y cambiar la relación entre universidad, Gobiernos y mercados.

La idea que buscamos demostrar en este trabajo es que en este proceso de desestructuración del viejo esquema y de estructuración de uno nuevo existe una importante línea de continuidad en términos de actores políticos y sociales que, ahora divididos y fragmentados, logran adecuarse y beneficiarse de las nuevas reglas de juego.

Para demostrarlo, el artículo se organiza en cuatro secciones. En la primera se define el concepto de reforma de la ES como parte de un proceso de ajuste estructural, iniciado en los años noventa —el cual se extiende hasta nuestros días-. En los apartados que siguen se describen las distintas etapas del proceso político, identificando los factores políticos — apoyos y restricciones endógenas y exógenasque contribuyeron a ella.

\section{El contexto regional de la reforma educativa: ¿por qué y para qué?}

Para responder a estas preguntas echaremos mano a dos bibliotecas muy diversas: la de la economía política que aporta elementos sobre el contexto de surgimiento y alcance de las reformas de ajuste estructural en América Latina por una parte, y la literatura específica del sector de la política educativa que se enfoca en el fenómeno de la internacionalización y las modalidades que adopta en el contexto de la globalización. 
Para ambas bibliotecas el punto de partida de esta reforma en América Latina es a mediados de los años noventa como correlato de las reformas estructurales. Se comprende como una reforma que reemplaza un modelo de desarrollo hacia adentro, promovida por el Estado por un nuevo patrón de desarrollo centrado en la inversión privada, la apertura externa y las señales del mercado. Con el cambio de época, también mudó el concepto de reforma estructural, que particularmente en los años sesenta aludía a los cambios del régimen de propiedad (como la reforma agraria) y que en los años ochenta pasó a interpretarse como la "reacción de las condiciones de acumulación privada y el fortalecimiento de la competitividad internacional” (Torre, 1999, p.12).

Las reformas estructurales de los años ochenta llegaron a América Latina para resolver una situación de fuerte adversidad económica, caracterizada como la crisis de la deuda externa. Debajo de su apariencia coyuntural se escondía el agotamiento del modelo de industrialización por sustitución de importaciones (ISI) que había sido puesto en marcha desde la posguerra. Este modelo de desarrollo industrial se apoyaba sobre la idea de un Estado "garantista", cuya estructura institucional emularía la idea de estado de bienestar de los países industrializados, que a diferencia de estos últimos careció de racionalidad. Su perfil institucional fue más el resultado acumulativo de cambios parciales introducidos por las crisis externas y las presiones de los distintos lobbies internos. Si bien el resultado fue un proceso de industrialización con modernización social que sin la intervención del Estado no hubiera sido posible, la apropiación de los recursos del Estado por los privados (corporaciones empresariales, sindicales, militares, etc.) terminaron por entorpecer la gestión y la capacidad decisoria de los gobiernos al limitar su autonomía y su coherencia interna por la acumulación de compromisos fiscales y de arreglos corporativos ${ }^{2}$.

\footnotetext{
2 La matriz Estadocéntrica se apoyó sobre políticas destinadas a proteger la industria nacional (barreras arancelarias, créditos subsidiarios, exenciones), a generar condiciones para el empleo (política aboral, negociaciones colectivas, infraestructura en servicios públicos) y a la inclusión social (acceso universal a la educación); pero sus bases de financiamiento no eran sólidas ni autosustentables en el tiempo. El financiamiento del ISI se apoyó sobre cuatro pilares: la apropiación de la renta de recursos naturales, la captura del ahorro privado a través de los fondos de seguridad social, los impuestos directos e indirectos y el endeudamiento externo (Cavarozzi, 1994 citado por Torre, 1998).
} 
Los libretos de los organismos financieros que salieron al rescate de América Latina tras la crisis de la deuda externa apuntaron en dos direcciones distintas y se sucedieron en el tiempo. El primero apuntó a la reforma de la macroeconomía con políticas de austeridad fiscal, privatizaciones, liberación comercial y desregulación económica. La segunda, en cambio, apuntó a sectores específicos del ámbito social y político como salud, justicia y educación, el cual ocupa nuestra atención en este trabajo. Si bien cada una de estas reformas tenía un objetivo específico, todas ellas convergían en una gran reforma que consistía en recortar los compromisos fiscales, así como la remoción de los arreglos corporativos para poder desvincular la gestión del Estado de las presiones del sistema político, y con ello recuperar los recursos públicos para abrir paso a un nuevo orden o modelo de desarrollo, centrado en el liderazgo de la inversión privada y los mercados.

El segundo interrogante pone el foco en los objetivos específicos de la reforma educativa. En esta dirección hacemos uso de la biblioteca de las políticas educativas, tomando los trabajos que hablan de la internacionalización de la ES como el objetivo de estas reformas estructurales. Para esta literatura, los procesos de internacionalización no son novedosos en las instituciones universitarias, sino concomitantes a su creación en el medioevo, de allí proviene su nombre ${ }^{3}$. Sin embargo, es a finales del siglo xx que el fenómeno de internacionalización se universaliza como respuesta a los procesos de globalización económica. En este sentido, es la globalización económica, entendida como la activación de flujos comerciales y financieros, acompañada por los avances tecnológicos los que empujan a la ES a incorporar elementos

\footnotetext{
3 Desde entonces, y hasta la actualidad, las IES desarrollan actividades de internacionalización como los programas de movilidad, la creación de redes de intercambio académico para mejorar su calidad y producción, etc. Primero fueron los Gobiernos, con vocación hegemónica como EE.UU. los que en los años cincuenta promovieron la internacionalización de sus IES como estrategia de política exterior para promover su cultura hacia terceros países y al mismo tiempo atraer "cerebros" de ellos. Una década más tarde, se sumaron los organismos internacionales, como la Unesco, que buscaron promover vínculos solidarios entre países centrales y los periféricos para promover el desarrollo.
} 
internacionales a su currículo y a sus estrategias institucionales para poder sobrevivir y mejorar su proyección global ${ }^{4}$.

Los consensos llegan hasta allí y las divergencias entre los autores surgen al momento de interpretar los alcances de la internacionalización y sus efectos. Para un grupo mayoritario de autores, la internacionalización es una respuesta de carácter propositivo frente a la globalización (de Wit, 2011; Tobón, Gallego \& Yepes, 2013; Sebastián, 2011, entre otros). En esta visión, las protagonistas de la reforma educativa son las IES, las cuales deciden de manera voluntaria qué tipo de estrategias de internacionalización adoptarán. Tradicionalmente las opciones han oscilado entre la cooperación o la competencia ${ }^{5}$, pero en tiempos de globalización la internacionalización supone además un proceso dual que combina dos dimensiones. Una dimensión interna, que se refiere al cambio en la cultura institucional, que consolida una nueva gama de valores en lo que respecta a la docencia, la investigación y la extensión universitaria, y una dimensión externa, que hace referencia a la proyección de las IES hacia el mundo, la cual supone la incorporación de prácticas de difusión y mercadeo. Si bien tanto la cooperación como el mercadeo aparecen como complementarios para la internacionalización, cada IES construye su propia estrategia con base en los recursos y prioridades de los que disponga y de los objetivos específicos que persiga. Aquellas instituciones que privilegian la dimensión interna, tendrán a la cooperación internacional

\footnotetext{
4 Estos elementos incluyen la creciente integración de la investigación, el uso del inglés como lingua franca para la comunicación científica y, de algún modo, para la educación superior a nivel mundial, la importancia cada vez mayor de un mercado laboral internacional para los académicos y científicos y, más particularmente, todos los aspectos relacionados con las TIC. Estas facilitan la comunicación, permiten mejorar el almacenamiento, la selección y la difusión del saber a todos los niveles y vuelven posible la oferta de programas académicos de todo tipo por medio de lo que se ha llamado el aprendizaje mediante redes electrónicas o e-learning (Altbach \& Knight, 2006, p. 14).

5 Uno de tipo cooperativo, que caracterizó los años 70 y 80, en el que prima la cooperación entre instituciones, el intercambio de conocimientos y asociaciones bilaterales entre instituciones. Y otro modelo competitivo, característico de los años 90 en adelante, enfocado hacia la competencia, la comercialización de la ES y el suministro transfronterizo de conocimientos. En este último, las estrategias a seguir se enfocan en la reformulación de planes de estudio, las innovaciones en el proceso de enseñanza y en la implementación de la movilidad estudiantil para lograr y explotar las ventajas comparativas de cada institución (de Wit, 2011).
} 
como su principal instrumento operativo; mientras que aquellas en las que se privilegia la dimensión externa, el instrumento a utilizar será la competencia a través de los rankings internacionales.

Para esta visión la internacionalización puede adoptar múltiples modalidades, entre las que se destacan el intercambio de profesores, investigadores y estudiantes, la formación y títulos en función del mercado global, la atracción de estudiantes extranjeros, la concreción de alianzas internacionales tanto en lo académico como en lo empresarial, el desarrollo de colaboraciones académicas y de investigación, el logro de acreditaciones internacionales y la apertura de campus universitarios en el exterior, entre otros. Por último, esta visión analiza el fenómeno de manera ingenua, bajo la premisa de que todas las IES y los países pueden aprovechar estas estrategias, en igualdad de condiciones. Solo hace la salvedad de que esta multiplicidad de modalidades de cooperación internacional estará presente en mayor o menor medida en las IES, de acuerdo al grado de sociabilidad de las mismas y al valor añadido por ellas (Sebastian, 2011, p. 8).

Para un segundo grupo de autores, la internacionalización genera y refuerza la desigual distribución de roles y de beneficios entre los países y regiones del mundo (Cordera Campos \& Santamaría Ambriz, 2008; Correia Lima \& Saraiva de Alburqueque, 2006). A diferencia del primer grupo, estos trabajos analizan los cambios introducidos por la globalización sobre los sistemas nacionales y sus efectos sobre las sociedades. Así por ejemplo, Correia Lima y Saraiva de Alburqueque (2006) focalizan su estudio sobre los flujos migratorios y señalan que los procesos de globalización económica han contribuido a profundizar las brechas que tradicionalmente separan a los países del centro de los de la periferia, generando un proceso de concentración de los activos científicos y tecnológicos en los primeros, y un efecto de reproducción del modelo global en los segundos, de la misma manera que ocurre en otros ámbitos del accionar público, como en la esfera militar y económica.

Reforzando esta idea de desigualdad dentro del mundo, señalan que frente a los mismos estímulos la capacidad de respuesta de los países industrializados fue mayor que la de los países periféricos, ya que si 
bien el estímulo es el mismo, en los primeros se concentra la mayor cantidad de capital académico. En materia de políticas de movilidad trasnacional, por tomar un ejemplo, los gobiernos del norte refuerzan sus políticas de movilidad "activa", atrayendo estudiantes de Asia y América Latina; mientras los países del sur, responden con políticas de movilidad "pasiva". Cabe destacar el avance en iniciativas entre países en desarrollo (sur-sur) las cuales se apoyan en algunas ventajas comparativas, como el idioma, a través del potenciamiento del turismo idiomático por ejemplo.

Dentro de este segundo grupo, se ubican los trabajos que estudian el proceso de reforma educativa que proponemos analizar en este trabajo. Sin embargo, a diferencia de nuestra propuesta, estos autores analizan el proceso de reforma como un acto administrativo en el que los gobiernos nacionales, frente a la urgencia y la desfavorable correlación de fuerzas entre los países acreedores, se apropiaron de las propuestas externas, introduciendo cambios menores que no alteraron el libreto neoliberal (González, 2007; Mollis, 2007; Noriega \& Montiel, 2014; Solanas, 2011; entre otros).

La propuesta de este trabajo es la de ampliar estos análisis en la profundidad y en el tiempo. Entendemos que estas reformas promercado no se agotaron con el fin del Consenso de Washington, sino que continuaron a través de distintos mecanismos y actores. De la misma manera, consideramos que la reforma educativa es una operación sustancialmente política cuyo desenlace depende de las salidas contingentes de las elites gubernamentales y de su capacidad para sumar apoyos en actores sociales e institucionales.

\section{Los alcances de la apertura unilateral: las reformas sectoriales del вм}

A principio de los años noventa, el gobierno de Carlos Menen (19891995) llevó adelante un profundo proceso de reforma del sistema de 
educación suprior ${ }^{6}$, que puso fin al modelo de autonomía universitaria: privatizó la oferta, descentralizó la gestión e incorporó un mecanismo de control de calidad (Chiroleu, 2003). El modelo de autonomía universitaria, que caracterizaba al sistema universitario argentino desde sus orígenes en 1918, estaba basado en tres principios: educación pública y laica, el autogobierno y el financiamiento estatal. La gratuidad de la enseñanza llegaría recién en 1949 con el gobierno de Perón. Este sistema había logrado poner a Argentina como uno de los países líderes en la región en términos de la cobertura y la calidad de la ES; no obstante, a partir de los años sesenta comenzó a dar señales de agotamiento como consecuencia del crecimiento de la matrícula, por un lado, y de la baja inversión pública, por el otro.

A las restricciones presupuestarias se sumaría un deterioro de la calidad educativa y de la investigación fruto de sucesivas intervenciones militares que se repitieron entre 1955 y 1983. Desde entonces, los gobiernos que se sucedieron, en su mayoría autoritarios, intentaron solucionar el problema de distintas formas, como por ejemplo mediante la apertura a la oferta privada y el ingreso restrictivo (mediante cupos). Sin embargo, ninguna de ellas pudo resolver el problema de fondo, por el contrario complicaron aún más el panorama (Ballerini, 2013).

En 1993, el Gobierno nacional avanzó hacia la reforma estructural del sector educativo de la mano de las ideas y préstamos de los bancos multilaterales de desarrollo ${ }^{7}$. La propuesta del BM justificaba la reforma por la necesidad de mejorar la "baja calidad" de la educación,

\footnotetext{
6 El sistema de ES argentino comprende a las universidades, los institutos universitarios e institutos de educación superior de gestión privada o estatal. En la actualidad está conformado por 53 universidades nacionales, 49 universidades privadas, 7 institutos universitarios estatales, 14 institutos universitarios privados, 6 universidades provinciales, 1 universidad extranjera y 1 universidad internacional.

7 Esta reforma quedo plasmada en la Ley 24195, conocida como Ley Federal de Educación, promulgada en 1993, que tuvo dentro de sus principales objetivos la reestructuración de todo el sistema de educación nacional, descentralizando desde el Estado hacia las provincias, la gestión de los distintos niveles de enseñanza primario, secundario y terciario no universitario (SNU). De esta manera, se establecieron las bases de un sistema de educación de tipo dual o binario, en el que mientras las instituciones del SNU quedarían bajo la jurisdicción provincial; las universidades — sistema universitario (SU) — se mantendría bajo jurisdicción nacional.
} 
estigmatizada como un problema común a todos los países de América Latina y en vía de desarrollo ${ }^{8}$. En sintonía con el Consenso de Washington, sus propuestas apuntaban en tres direcciones. La primera consistía en abrir la oferta a los proveedores privados y diversificar las fuentes de financiamiento de las instituciones públicas. A su vez, proponía descentralizar la gestión universitaria, dándole más autonomía y poder a las instituciones subnacionales en la administración de sus propios presupuestos, en las contrataciones y en los despidos del personal. Por último, proponía controlar la calidad de la educación de grado y postgrado a través de agencias para la evaluación y acreditación como un ente autónomo del gobierno de turno, aunque financiadas por el Estado (Mollis, 2007, p. 79).

Sin embargo, los avances de estas reformas fueron dispares entre los países de América Latina. En el caso específico de Argentina, las políticas implementadas fueron el resultado de un proceso de negociación y de redistribución de poder entre los distintos actores universitarios y extrauniversitarios. La Ley de Educación Superior (LES), sancionada en 1995, ejemplifica muy bien la dinámica de este proceso político de reforma. La iniciativa provino del poder ejecutivo, motorizada por un equipo de tecnócratas reunidos en la flamante Secretaría de Políticas Universitarias (SPU).

Luego de suscribir un acuerdo con el BM en 1994, la sPU creó la Comisión de Acreditación de Postgrado (CAP) y puso en marcha la primera experiencia de evaluación, como requisito para poder acceder a subsidios del nuevo Fondo de Mejoramiento de la Calidad Educativa (FOMEC). Con posterioridad, el Gobierno envía el proyecto de ley al Congreso para reemplazar la CAP por una Comisión Nacional de Evaluación y Acreditación Universitaria (CONEAU) en la que además de postgrados se evaluaría la calidad de la oferta universitaria de

\footnotetext{
8 El descenso de la calidad académica no solo fue el resultado de la expansión de la matrícula y una inadecuada provisión de recursos financieros, sino que estuvo agravada por la ineficiencia en la utilización de los recursos existentes y la inequidad que supone la gratuidad de los estudios universitarios por una mayoría de estudiantes provenientes de familias acomodadas, lo cual tiene un efecto negativo en la distribución de los ingresos (Banco Mundial, 1994 citado por Chiroleu, 2003).
} 
grado. El proyecto de ley encontró la resistencia de las universidades públicas y privadas por considerar que, por una parte, amenazaba el principio de autonomía universitaria de muy diversas maneras ${ }^{9}, \mathrm{y}$ por la otra no resolvía los problemas presupuestarios ni garantizaba la gratuidad de la enseñanza. La resistencia a la LES se concentró principalmente en dos actores, la Universidad de Buenos Aires (UBA) - la más poderosa y masiva de todo el país — y las organizaciones estudiantiles, hegemonizadas por Franja Morada, brazo estudiantil del partido Unión Cívica Radical.

No obstante, la ley fue finalmente aprobada por el Congreso argentino. Las razones de este éxito se encuentran en las debilidades y grietas de la resistencia. Por un lado, la oposición estudiantil se redujo a la consigna y pelea por la gratuidad bajo el slogan del "no arancelamiento". Por otro, las IES se dividieron entre aquellas que se resistían a la intervención en todas sus formas, mientras otras reconocían la necesidad de mejorar los niveles y criterios de calidad bajo normas internacionales, para no perder sus clientelas cautivas en manos de la competencia privada o del exterior ${ }^{10}$. El principal apoyo a la iniciativa de reforma del gobierno provino de las nuevas universidades nacionales, que fueron creadas a partir de 1993, en el conurbano bonaerense y en otras regiones del país, en línea con el mandato de descentralización propuesto por el BM. En virtud de su reciente creación, estas nuevas IES tenían menos restricciones institucionales e intereses específicos que defender, además de estar tuteladas por el poder ejecutivo, encargado de nombrar al Rector Organizador, lo que hizo "posible pensar que el gobierno vio en ellas una oportunidad

\footnotetext{
9 En primer lugar, supeditaba los estatutos universitarios a la aprobación del Ministerio de Educación. En segundo lugar, autorizaba la intervención a las universidades por distintos motivos. En tercer lugar, facultaba al Ministerio a fijar los contenidos mínimos de los planes de estudio. En cuarto lugar, colocaba a la universidad pública en un lugar secundario en el sistema de evaluación y acreditación universitaria; en quinto lugar, fijaba criterios mínimos de regularidad hasta entonces privativos de las universidades. En sexto lugar, establecía criterios y condiciones para la composición de los órganos colegiados del gobierno universitario (Solanas, 2011, p. 184).

10 Entre las universidades nacionales que se resistieron a este proceso de acreditación se encuentran la Facultad de Ingeniería de la UBA y la de Ciencias Sociales, entre otras (entrevista con funcionaria de CONEAU).
} 
clara de encontrar interlocutores adecuados para implementar las reformas impulsadas" (Noriega \& Montiel, 2014, p. 169).

Para incentivar el apoyo de las universidades a la reforma, la LES estableció que en principio la acreditación sería voluntaria y que además estaría atada a la competencia de fondos públicos para mejorar la calidad y el desarrollo institucional. Los fondos creados para tal fin fueron dos: el Fondo para el Mejoramiento de la Calidad Universitaria (financiado en un $70 \%$ por el BM) y el Programa de Incentivos Docentes, restringido a las universidades públicas, para promover la investigación entre sus docentes (Marquis, 1998). A esos incentivos monetarios se sumaron los espacios de participación y decisión tanto de los rectores de las universidades públicas (Consejo Interuniversitario Nacional) como de las privadas - Consejo de rectores de Universidades Privadas (CRUP) — en los consejos regionales de ES, encargados de la planificación del sistema, $\mathrm{y}$ de representantes en minoría en la misma CONEAU ${ }^{11}$.

La LES fue aprobada en 1995. Si bien el sistema de acreditación y evaluación nacional fue establecido, otras reformas no llegaron a buen puerto, demostrando que en efecto se trataba de una negociación. Así, por ejemplo, las iniciativas de quitar la responsabilidad financiera del Estado en la universidad pública, de reducir personal y participar en la negociación con el cuerpo docente fueron neutralizadas por la resistencia de la universidad pública (Solanas, 2011). Las IES mantuvieron autonomía en la dirección de sus claustros y reforzaron la obligación del Estado de financiar la educación pública de grado. Solo se avanzó en la idea de tercerizar algunos servicios, como la limpieza y seguridad y de incorporar fuentes alternativas de financiamiento (pago de aranceles y contratación privada) para los cursos de posgrado.

\footnotetext{
11 La CONEAU, en líneas generales, se ocupa de realizar evaluaciones externas, de recomendar la acreditación de proyectos institucionales, de acreditar carreras de interés público y todas las careras de grado y posgrado. Está compuesta por 12 miembros designados por el poder ejecutivo a partir de las propuestas de los siguientes organismos: la Cámara de Diputados (3 postulaciones), la Cámara de Senadores ( 3 postulaciones), el CIN ( 3 postulaciones), el CRUP (1 postulación), la Academia Nacional de Educación (1 postulación) y el Ministerio de Educación (1 postulación). Cada uno de ellos dura 4 años en sus funciones, que pueden renovarse de manera parcial, pero todos ellos deben ser de reconocida trayectoria académica.
} 
Si bien las innovaciones avanzaron en distintas direcciones - ampliación de las fuentes de financiamiento, regulaciones nacionales, participación de los privados, cooperación internacional, vínculo con el sector productivo, sistema de rendición de cuentas y flexibilización curricular - la acreditación de calidad se constituyó en el principal nudo de las reformas unilaterales en Argentina.

Hacia el final de este periodo (1993-2003) se había producido una reestructuración profunda del sistema educativo argentino, en la que la relación entre la universidad, el Estado y el mercado era muy distinta a la de las décadas previas. No solo se habían redefinido las competencias sino que se habían incorporado una serie de nuevos actores con roles claves como la SPU, la CONEAU, los consejos regionales y muchas nuevas IES, tanto privadas como públicas. Esta reforma tambien ayudó a crear condiciones favorables para la internacionalización del sistema y de las IES de manera indirecta ${ }^{12}$. A su vez las universidades — tanto públicas como privadas - se vieron impulsadas a cambiar su tradicional lógica de cooperación por otra de competencia para acceder a los recursos públicos y para funcionar dentro de las reglas del mercado. El salto del mercado doméstico al internacional debió esperar unos diez años y fue, como analizaremos en el apartado siguiente, fruto de la apertura de negociaciones multilaterales de servicios educativos en la reunión de la OMC en Cancún en 2003.

\section{Los intentos de la apertura multilateral: la омс y el acuerdo birregional}

En términos de contenido, la propuesta de la OMC era ideológica y materialmente compatible con las reformas unilaterales implementadas desde el BM. Ambas instituciones globales — financiera la primera y comercial la segunda- evaluaban los resultados de la ES en

\footnotetext{
12 Entre estas condiciones se encuentra no solo la obligatoriedad de cumplir con los procesos de acreditación de acuerdo a parámetros internacionales (evaluaciones regulares con pares internacionales), sino también la posgraduación, que habilita a las IES a atraer estudiantes internacionales.
} 
términos de inversión y rentabilidad, y partían del diagnóstico de que el principal problema de la ES era el descenso de la calidad educativa fruto de un aumento de la matricula (demanda) y la falta de inversión pública. Para ambas instituciones la solución pasaba por abrir la oferta educativa al sector privado, su diferenciación radica en los alcances del rol del Estado y en el alcance de la apertura. Mientas el BM hablaba de abrir la competencia a los prestadores nacionales (IES privadas) y fortalecer el rol regulador del Estado, la OMC proponía ampliar esta apertura a la oferta internacional y asegurar la desregulación de la normativa nacional para permitir el acceso y el "trato nacional" a los prestadores internacionales ${ }^{13}$. Una visión global distinta a la del BM y la OMC fue la de la Unesco. Si bien este organismo internacional compartía el diagnóstico del problema acerca de la baja calidad y de las restricciones presupuestarias, señalaba que esto se daba en un marco de desigualdad en el acceso y diversificación de las instituciones, aspectos sobre los que se debía trabajar. Para ello, la solución consistía en más inversión social, económica y cultural para mejorar la competitividad económica y la cohesión social a través del apoyo público $^{14}$ (Chiroleu, 2000).

Con la firma del Acuerdo General de Comercio y Tarifas en Servicios (GATT, por su sigla en inglés) en la OMC, en 1995, se inició una escalada en el mundo a favor de la desregulación de las normativas nacionales en materia de compra y venta de servicios educativos ${ }^{15}$. La primera avanzada estuvo motorizada por los países anglófonos - EE.UU., Australia, Nueva Zelanda-, Suiza y Japón en el marco de la OMC pero no logró avanzar por la resistencia de los países desarrollados

\footnotetext{
13 La OMC justifica la necesidad de los Estados de ajustarse a una norma legal en el hecho de que por existir proveedores privados en los sistemas nacionales de educación, el arbitraje de los conflictos no podía estar en manos del Estado que era parte interesada, sino en reglas de control y arbitraje internacional.

14 Documento de Política para el Cambio y el Desarrollo de la Educación Superior (Unesco, 2/95).

15 Cuando la OMC habla de liberalizar el comercio de servicios, se refiere a la decisión de los Estados de resignar su derecho al uso de normativas que protejan a los prestadores nacionales frente a los extranjeros, permitiéndoles el libre acceso en su territorio y un trato equivalente al que reciben los prestadores nacionales.
} 
que entendieron que esta propuesta constituía una amenaza para sus propios sistemas en términos de la pérdida de contendidos curriculares y de estudiantes nacionales o extranjeros que optarían por propuestas más afines a las demandas del mercado laboral ${ }^{16}$. No obstante, la OMC fue exitosa en otras dos direcciones. Por una parte, instaló en el debate público la idea de que la educación superior no era solo un derecho sino un capital social que podía comprarse y venderse para promover el desarrollo de los mercados y la productividad. En esta batalla ideológica, moldeó y subordinó los discursos y las prácticas de otras organizaciones internacionales, que hasta entonces habían sostenido visiones alternativas y/o se mostraban respetuosas de la diversidad naciona ${ }^{17}$. Por otra parte, la OMC amplió las modalidades de compra y venta de servicios a nuevas formas de intercambio habilitadas por los nuevos desarrollos tecnológicos (las TIC) ${ }^{18}$.

La segunda avanzada en favor de la apertura multilateral la llevaron adelante los países desarrollados a través de la firma de acuerdos de libre comercio, donde la liberalización de los servicios educativos era parte constitutiva de ellos. Esta estrategia alcanzó a los países de América Latina y estuvo liderada por los EE.UU. con la propuesta

16 Entre 1995 y 2002, para tomar un ejemplo, de los 186 países miembros tan solo 32 habían contraído algún compromiso concreto en materia de educación postsecundaria y solo dos eran latinoamericanos.

17 Este es el caso de la Unesco y la OCDE, que hacia finales de los 90 empezaron a defender la idea y/o necesidad de homogeneizar los programas y los currículos nacionales a nivel global a través de políticas de acreditación y/o criterios de evaluación de calidad construidas a partir de indicadores preestablecidos e inspirados generalmente en universidades de países desarrollados. Lo mismo ocurrió con las llamadas agencias de la cooperación intra o interregional, que encargadas de la evaluación y aseguramiento de la calidad, terminaron por contratar prestadores privados, que persiguieron el lucro contribuyendo así al recrudecimiento del comercio educativo (Didou Aupetit, 2005, p. 14).

18 En esta dirección, el GATT tipifica y pone en un catálogo a la oferta transfronteriza que incluye al e-learning, que no implica la necesidad del movimiento físico del consumidor o proveedor; el consumo en el extranjero: en el que el consumidor se desplaza al lugar del proveedor (movilidad de estudiantes); la presencia comercial: donde el proveedor educativo se establece en otro país para ofrecer sus servicios (incluye los campus satélites y asociaciones con instituciones locales), y por último la presencia de personas naturales: corresponde a aquellos que viajan de un lugar a otro a ofrecer servicios educativos de forma temporal (como profesores, investigadores, etc.). 
de un Área de Libre Comercio de las Américas (ALCA), y la Unión Europea a través de acuerdos bilaterales o birregionales. Si bien en algunos casos finalizaron con éxito los acuerdos bilaterales - Chile (2003), Colombia (2004), Costa Rica (2004), Perú (2004) - con EE.UU. y/o Europa; en el caso de Argentina la negociación se canalizó de manera regional a través del Mercosur. De entre estas dos propuestas, la que más avanzó fue la de la UE que, a diferencia de la de EE.UU., estuvo acompañada por ayuda financiera y técnica. A pesar del rechazo de las IES públicas y privadas a la incorporación de los servicios educativos, las negociaciones avanzaron hasta 2002. En esta dirección, los países del Mercosur firmaron el Protocolo de Montevideo (2007) en el que se comprometían a liberalizar el comercio de servicios intrazona en un plazo de diez años para llegar juntos al mercado europeo. Sin embargo, la letra del acuerdo nunca se puso en marcha por dos razones fundamentales: la pérdida de dinamismo de las negociaciones a medida que se avanzaba en la exigencia de eliminar las barreras agrícolas, y la resistencia que la idea de apertura regional generó en gran parte de las universidades nacionales, tanto públicas como privadas. Finalmente la negociación birregional se detuvo y el protocolo de servicios de Mercosur nunca se internalizó (Botto \& Peixoto, 2007).

La tercera y última avanzada en favor de la apertura multilateral tuvo como protagonista a las empresas que venden servicios por fuera de sus Estados (transnacionalización). El avance de este comercio de servicios transfronterizo en América Latina fue el resultado de la debilidad regulatoria de los Estados y la ausencia de mecanismos de control y acreditación de calidad para impedir el avance de la oferta internacional, más que de la fortaleza de los proveedores para cambiar las reglas del juego. La oferta transnacional de servicios educativos ha adoptado tradicionalmente tres formatos: los esquemas de coinversión de programas de internacionalización, la oferta de carreras y la constitución de organismos especializados en la venta de servicios. Con la globalización, la oferta se ha ido ampliando de cursos de posgrados a licenciaturas, carreras cortas de dos años o actualización profesional. En términos de proveedores, el mercado de la transnacionalización en América Latina se divide en dos: los proveedores 
externos y los proveedores latinoamericanos. Entre estos últimos, los proveedores se han ido ampliando con el tiempo, pasando de la oferta privada a la oferta pública en virtud de la cercanía idiomática y cultural que los favorece ${ }^{19}$.

Siguiendo a Didou Aupetit (2006), entre los principales problemas que potencian el avance indiscriminado de este comercio transfronterizo en la región se encuentran la diversidad normativa que separa a los distintos países ${ }^{20}$, así como el bajo grado de involucramiento efectivo de las IES para construir acciones tendientes a la convergencia regional, la existencia de dispositivos de créditos y de reconocimiento de titulos que son disfuncionales en cuanto a la legibilidad, funcionamiento interno y eficiencia; la ausencia de confianza recíproca entre los países y entre sus propias IES, y la ausencia o debilidad de dispositivos para actualizar las normas y la falta de recursos y/o voluntad para crear centros de acopio de información.

En gran medida, el caso Argentino se escapa de esta regla. El avance de los servicios transfronterizos ha sido menor y ello se explica, en gran medida por la presencia de la CONEAU que ha sido exitosa para cuantificar y controlar el aumento indiscriminado de la oferta. El sistema también cuenta con una normativa especial y restrictiva para el ingreso

19 Así por ejemplo, en un primer momento, la oferta de servicios provino de actores privados externos, que a manera de iniciativas individuales o de red (consorcios ente universidades o con empresas) ofrecían carreras de posgrado o licenciaturas en carreras cortas vinculadas con la formación de profesionales. Con posterioridad, fueron los gobiernos los que asumieron la iniciativa, promoviendo la inversión externa privada y la venta de servicios educativos hacia otros países de la región, aprovechando la cercanía geográfica. Sus modalidades variaron desde la instalación de oficinas de enlace en otros países — como hizo la Universidad de Chile en Washington o la Universidad Andina Simón Bolívar en Bogotá- a la convocatoria de capitales a sus propios países con proyectos de coinversión — como la Universidad de las Américas en Uruguay-; o la misma promoción de sus IES a través de la creación de comités de servicios educativos en sus embajadas, como hace Chile desde 2002 (González, 2007).

20 Miranda (2008) tipifica esta variedad en seis modalidades: hay países que prohíben la oferta, como la Republica Dominicana; otros donde no está legislado el acceso, como Chile, Perú y América Central; otros en donde se las somete al mismo régimen legal que las privadas nacionales, como en Bolivia; países donde los proveedores foráneos están sujetos a un régimen especial, como en Argentina, y por último, países como Colombia donde están obligadas a asociarse a instituciones nacionales de educación superior o funcionar sin ánimo de lucro. 
de proveedores externos, con la única excepción de la Universidad de Bolonia (Decreto 276/99). A pesar de ello, si bien estos dispositivos han sido exitosos para controlar la oferta y la demanda formal, su capacidad para frenar la venta de servicios y las modalidades más informales - como los cursos a distancia, asociaciones y proyectos de inversión conjunta - es menor y está por fuera de todo registro.

Una manera de reducir estas dificultades nacionales es a través de los procesos de integración y de cooperación regional. En el apartado que sigue, analizaremos los alcances de los programas del Mercosur al respecto.

\section{La cooperación regional: Mercosur}

A diferencia de la apertura unilateral y multilateral, la inclusión de la agenda de ES en el ámbito del Mercosur fue una decisión consensuada entre los Gobiernos nacionales y no una imposición desde afuera. La iniciativa partió de los ministros de educación en su afán de frenar el entusiasmo de algunos funcionarios de las cancillerías por avanzar en un acuerdo birregional con la Unión Europea por una parte; así como de promover acciones conjuntas para frenar la entrada de servicios trasfronterizos de baja calidad (ver apartado anterior). La propuesta consistía en crear un espacio de cooperación solidaria de alcance regional para el intercambio de recursos y la coordinación de acciones entre los Estados del Mercosur ampliado. En la construcción y en la implementación de esta política de apertura regional, Argentina desempeñó un papel importante, que se ha ido ampliando desde el año 2003 con la decisión de los gobiernos de Néstor y Cristina Kirchner de convertir la educación superior en el eje de su política exterior y al Mercosur en el ámbito prioritario de su estrategia de cooperación multilateral ${ }^{21}$.

\footnotetext{
21 Esta estrategia se orientaba en cuatro direcciones: i) buscar el liderazgo regional a través de la asistencia técnica en materia de acreditación de carreras; ii) dirigir los recursos financieros y técnicos hacia el Mercosur/UNASUR teniendo como principales socios de cooperación a Brasil y los países de América Latina; iii) orientar la integración y convergencia de los sistemas nacionales a través de los principios de cooperación solidaria y respeto de la soberanía institucional; iv) promover la cooperación interuniversitaria y asegurar así la
} 
Con base en estos objetivos, la política de ES del Mercosur se orientó en tres direcciones: i) crear un sistema regional de acreditación de calidad de carácter permanente, vinculante y aplicable a todos los niveles de enseñanza universitaria (grado, postrado y tecnicaturas); ii) promover la movilidad académica de estudiantes y profesores entre los países de la región para fortalecer la cohesión social y la idea de identidad regional, y iii) promover la cooperación entre las IES regionales para favorecer la mejora de la calidad a través de programas de enseñanza, investigación y docencia conjuntos.

De estas tres políticas, la que más avanzó fue la de la acreditación y lo hizo a través de dos programas sucesivos. La primera experiencia piloto - llamada Mecanismos de Acreditación para carreras de grado (MEXA) - tuvo lugar entre 2002 y 2008 y se aplicó a las carreras de ingeniería y medicina. El segundo programa —el Sistema de Acreditación Regional de Carreras Universitarias (ARCU-sur/Mercosur)— se inició en 2009, incorporó las carreras de arquitectura, veterinaria, odontología y economía. El propósito de sendos programas fue el de evaluar y acreditar la calidad de la oferta regional adjudicando un sello de calidad Mercosur a las mejores universidades en cada campo de estudio. La dinámica del proceso reprodujo a nivel regional lo que Argentina venía implementando a nivel nacional a través de la CONEAU, es decir, generar espacios y oportunidades de diálogo e intercambio entre académicos, técnicos y funcionarios para ayudar a las IES a alcanzar una mejor calidad ${ }^{22}$. Estos dispositivos de acreditación regional apuntaban a mejorar las capacidades de dos actores.

inserción estratégica del sistema universitario por medio de políticas de movilidad (Larrea \& Astur, 2014).

22 El sello sería otorgado por la Reunión de Agencias Acreditadoras del Mercosur (RANA) y creado de manera ad hoc por expertos nacionales e internacionales en el tema. Estos expertos evaluarían el perfil curricular, la calidad de los docentes y el número de egresados, entre otras consideraciones de cada IES y adjudicarían el sello en la medida en que se aproximara a los criterios convenidos de calidad. No se buscaba imponer un modelo sino más bien promover redes de confianza y de reconocimiento mutuo entre las distintas instituciones de la región. El costo sería afrontado por cada institución académica, ya que se esperaba que fueran las universidades las que tuvieran interés en adquirir el sello Mercosur, para poder competir en mejores condiciones en el mercado regional y atraer más estudiantes y recursos financieros y de cooperación técnica. 
Por una parte, de los Estados miembros del Mercosur que carecían de instituciones y mecanismo de acreditación de calidad, proveyéndoles de asistencia técnica y de recursos financieros provenientes de los países con experiencia en la materia. Por otra parte, de las IES públicas y privadas de la región, proveyéndolas de herramientas y oportunidades para mejorar su estrategia de internacionalización dentro y fuera de la región. En esa dirección se orientaron también los programas de movilidad del Mercosur, cuyos beneficios estuvieron inicialmente reducidos a aquellas carreras que contaran con la acreditación regional; a través de ellos se buscaba promover la creación de redes entre las comunidades académica, de la confianza y del reconocimiento mutuo entre las IES de los distintos países del Mercosur. Con el tiempo, las iniciativas de movilidad se fueron ampliando con el aporte de otros actores influyentes del sistema que, como el Grupo Montevideo, se sumaron a estas estrategias de internacionalización competitiva.

Si bien los resultados de este proceso de acreditación/movilidad regional no fueron significativos en términos del número de carreras acreditadas y de movilidad, su principal contribución es de tipo cualitativo y se manifiesta en el acopio de la información, la construcción de reconocimiento mutuo entre sistemas que desconfiaban unos de otros hasta entonces y los avances en la definición de parámetros de calidad en algunas carreras $^{23}$. Sobre todo, este proceso contribuyó a fortalecer las capacidades técnicas e institucionales nacionales de aquellos países que no habían logrado avanzar en este aspecto de las reformas unilaterales promovidas por el BM. En este sentido, la acción coordinada de la RANA (Red de Agencias Nacionales de Acreditación) ayudó a crear instituciones y mecanismos de acreditación nacional en países que en los que - como Bolivia, Uruguay, Paraguay, Ecuador y Venezuela— no existían o no estaban en funcionamiento ${ }^{24}$. El Mercosur Educativo también ayudó a

\footnotetext{
23 Entre los acuerdos firmados está el Protocolo de Integración Educativa sobre Reconocimiento de Títulos Universitarios para la Prosecución de Estudios de Posgrado en las Universidades de los países del Mercosur, que posibilitaría también el reconocimiento posterior del títulos de posgrado obtenido, y el Protocolo de Integración Educativa para la Formación de Recursos Humanos a nivel de posgrado en los países del Mercosur (Acta 02/95 RME).

24 Este patrón de cooperación sur-sur se mantiene en la actualidad con una dinámica bilateral liderada por Argentina, que se ha ido ampliando a Ecuador y Venezuela. Con la
} 
consolidar los procesos de internacionalización interna y externa, promoviendo los procesos internos de actualización curricular, la creación de redes de intercambio docente y de investigación que potenciaron las oportunidades de proyección internacional, atracción de estudiantes y firma de convenios internacionales. Sin embargo, estas oportunidades no se distribuyeron de manera equitativa sino que beneficiaron más a aquellas instituciones públicas de carreras profesionales, que supieron adecuar sus necesidades a las demandas del contexto interno y externo desde el principio del proceso de reforma.

\section{Conclusiones}

Después de reconstruir la trayectoria de las reforma de la ES en Argentina, estamos en condiciones de caracterizar el comportamiento de los principales actores exógenos y endógenos que participaron en ella y sus efectos sobre el resultado de la misma, que de manera ineludible se fue apartando del libreto originalmente planteado por los organismos financieros internacionales en el Consenso de Washington.

Tomamos como punto de partida el quiebre de un modelo de educación garantista, apoyado en la idea de acceso universal a la educación superior de calidad garantizado por el financiamiento público y la gestión autónoma por parte de la comunidad académica. Este modelo, como señalamos en la introducción, entró en crisis en los años sesenta por la irrupción militar de Onganía (1966-1970), su intervención en la autonomía universitaria y el desfinanciamiento de la universidad pública en relación inversa al aumento de la matriculación. Hacia mediados de los años ochenta, la situación de desfinanciamiento estructural del Estado, no privativa de esta área sino del modelo de desarrollo, entró en crisis terminal. En el caso de la educación universitaria, las escasas intervenciones del gobierno democrático de

creación del Consejo Sudamericano de Educación Cultura, Ciencia y Tecnología (COCECTI), los avances dados en el Mercosur se expanden a todos los países miembros de la UNASUR provistos por técnicos y funcionarios argentinos (Entrevista a funcionario del ministerio de educación, noviembre 2015). 
Alfonsín (1993-1989) terminaron por agravar la situación ${ }^{25}$. El sistema mostraba tres graves falencias: i) el florecimiento a lo largo del tiempo de múltiples formas de corporatismo con fondos públicos a través de asignaciones específicas — como el Ciclo Básico Común-; ii) el crecimiento de un aparato estatal (burocracia) con escasa capacidad decisoria, y iii) la pérdida de calidad académica y de investigación científica y técnica.

En este contexto nacional, caracterizado por la pérdida de autonomía y de coherencia interna del Estado, es en el que las ideas de transformación hacia el mercado encuentran un terreno fértil para su puesta en marcha. La primera conclusión a la que llega el trabajo es que no se trató de un movimiento acotado en el tiempo, sino de una sucesión de etapas. El primer paquete de reformas sectoriales llegó de la mano del BM, alineado a los preceptos generales del Consenso de Washington. La propuesta del BM reproducía en el ámbito de la educación superior el libreto de las reformas económicas. Se hablaba de la necesidad de recortar los compromisos fiscales de parte de los Gobiernos en materia de educación superior; se proponía remover los arreglos corporatistas con el objetivo de desvincular la gestión del Estado de las presiones del sistema político, y por último se hablaba de sentar las bases de un nuevo modelo de desarrollo, centrado en el liderazgo de la inversión privada y el mercado educativo global. La segunda reforma global llegó a América Latina de la mano de la OMC de manera más o mensos simultánea.

Con la apertura de las negociaciones multilaterales de servicios a nivel global en 1994, la OMC — con el auspicio de las empresas y Gobiernos de países anglófonos - buscaba generar nuevas y mayores oportunidades legales para el acceso de la oferta/inversión privada transnacional en el mercado educativo de los países en desarrollo.

\footnotetext{
25 En los años 80, en gran medida como resultado de la política de ingreso irrestricto, el número de alumnos se multiplicó. La matrícula paso de 275000 estudiantes en los años 70 a más de 2,25 millones en 2010. A contrapelo de esta tendencia de la demanda, los niveles de inversión y financiamiento se redujeron, encontrando su mínimo nivel histórico con la hiperinflación de 1989 (García Solá, 2004).
} 
En este sentido, esta reforma no se oponía a la primera oleada de apertura, por el contrario la complementaba y ampliaba sus alcances. Por último, la tercera etapa de reformas vino de la mano de la Unión Europea, que a través de incentivos materiales e ideológicos, buscaba promover en el mundo la creación de un mercado regional de educación, en el que los países del bloque intentaran coordinar sus políticas nacionales en materia de ES para favorecer la creación de una área de libre movimiento de personas con fines académicos. La motivación que tenía la UE de transferir su modelo de gobernanza educativa a todo el mundo no solo estaba motorizada por principios solidarios sino también por el interés económico de atraer estudiantes extranjeros y evitar el éxodo de los propios.

La segunda conclusión que demuestra este recorrido del proceso de reforma es que en ningún caso el libreto global fue implementado al pie de la letra. Por el contrario, fueron filtrados por las orientaciones ideológicas y los cálculos políticos del presidente de turno. En el caso particular de Menem (1989-1998) sus preferencias pasaron principalmente por licuar el poder de la universidad pública de Buenos Aires, históricamente en manos del partido de la oposición (la Unión Cívica Radical) creando nuevas universidades en el conurbano bonaerense. También aprovechó para reestructurar el sistema nacional de ES, descentralizando la gestión presupuestaria y administrativa a las provincias y por último, pero no por ello menos importante, abrió la oferta al sector privado para promover la competencia, no sin antes recuperar para el Estado la capacidad de regular la calidad de la oferta a través de la CONEAU.

A diferencia de la primera reforma, la de la apertura multilateral propuesta por la OMC no prosperó en la gestión menemista. Si bien entró en la agenda de los funcionarios de la cancillería, quienes tuvieron expresas indicaciones de avanzar en la negociación normativa que favoreciera una mejora en las posiciones nacionales en la agenda agrícola (como moneda de intercambio), su avance se vio frenado por la oposición del Ministerio de Educación. En este tema en particular, el Ministerio de Educación se hacía eco de la resistencia de los rectores de las universidades públicas y privadas, que se oponían a refrendar sus titulos con la 
oferta internacional, reservándose para ellos la posibilidad de proteger el mercado nacional a través de barreras normativas a la inversión privada en el espacio nacional.

La respuesta definitiva al comercio de servicios transfronterizos, sin embargo, tuvo que esperar a la tercera etapa de reformas estructurales: la caracterizada en el trabajo como de regionalización. Este tercer libreto global de reformas educativas en América Latina fue apropiado por el Gobierno argentino, en este caso en las gestiones de Néstor y Cristina Kirchner (2003-2015). Para sendas gestiones la integración regional en general y en educación superior y ciencia y tecnología en particular se convirtió en el eje de su política exterior y ocupó un papel central en su proyección regional como líder de la cooperación bilateral, ayudando a los países del bloque y de la UNASUR a crear capacidades institucionales y técnicas para la construcción de agencias de acreditación a nivel nacional. Hacia adentro, la coordinación de políticas de acreditación regional de movilidad generaría incentivos para que las universidades, como la de agronomía, se convencieran de la necesidad de acreditar su calidad bajo la CONEAU para así posicionarse en el mercado internacional y proveerse de recursos alternativos provenientes del exterior.

La tercera conclusión que se levanta de la experiencia argentina habla de las resistencias políticas con que toda reforma se enfrenta y la necesidad de las elites gubernamentales de movilizar apoyos sociales y capacidades institucionales para llevarlas a cabo. De entre todas las resistencias que enfrentó la reforma del menemismo, el rechazo de la UBA a la creación de la CONEAU fue sin duda el más importante. No solo porque se trataba de un actor político y social de relevancia por su peso histórico y sus dimensiones, sino porque el objetivo de crear una instancia de control académico atacaba el principio histórico de la autonomía universitaria. Sin embargo, lejos de ser taxativo y homogéneo, este rechazo se caracterizó por divisiones internas que mostraron una gran capacidad del Gobierno de diluir la oposición a través de una hábil política de incentivos financieros (incentivos docentes a la producción, apertura de nuevas ventanillas de financiamiento a través de cursos de posgrado) y políticos, como la creación de instancias 
decisorias superiores. Los principales detractores fueron los decanos de las universidades de ciencias sociales, de humanidades y de agronomía, que rechazaron entrar en los procesos de evaluación de calidad.

Por último, el caso argentino permite extraer alguna referencia en torno a los efectos sociales y políticos que provoca el proceso de desmantelamiento del sistema garantista de ES y su reemplazo por otro afín a las reglas de la competencia y del mercado. La redistribución de los recursos de poder liberados por el desmantelamiento se realiza de manera desigual entre los actores del sistema, que genera dos efectos innovadores. En primer lugar, acrecienta las diferencias preexistentes de calidad y de influencia entre las universidades públicas. Este fenómeno se puso en evidencia entre las facultades de carreras profesionales que se adecuaron rápidamente a las nuevas reglas de la evaluación y la competencia, y las facultades de ciencias sociales y humanidades que se resistieron a incluirse en el nuevo sistema, y en consecuencia perdieron acceso a recursos. En segundo lugar, aumenta la capacidad de negociación de las instituciones privadas con posibilidades de convertir las nuevas reglas de juego en la expresión institucional de relaciones de fuerza que les son favorables. Sin ir más lejos y a manera de ilustración, las facultades privadas de ciencias sociales sacan provecho del espacio vacante en el mercado y, haciendo uso del conocimiento externo que les ofrecen sus aceitadas redes internacionales, ofrecen una propuesta más atractiva para los sectores de mayor poder adquisitivo.

\section{Referencias}

Altbach, Philip G., \& Knight, Jane. (2006). Visión panorámica de la internacionalización en la educación superior: motivaciones y realidades. Perfiles Educativos, 28, 13-39.

Ballerini, V. (2013). La internacionalización de la educación superior en la Argentina (Tesis de maestría, Facultad Latinoamericana de Ciencias Sociales -Flacso-, Argentina).

Botto, M. (2015). La transnacionalización de la educación superior ¿Qué papel juegan los nuevos regionalismos en la difusión de estas ideas? El caso del Mercosur (1992-1912) en perspectiva comparada. Revista Iberoamericana de Educación Superior (RIES), 6(16), 90-108. 
Botto, M., \& Peixoto Batista, J. (2007) La incidencia de la academia en las negociaciones de los servicios de salud y educación en la Argentina: desafíos y oportunidades. En M. Botto, (Coord.), Saber y politica en América Latina (pp. 247-291). Buenos Aires: Prometeo Libros.

Chiroleu, A. (2003) La educación superior en los países del Mercosur. El reconocimiento de las singularidades como punto de partida de la integración regional. Revista Perfiles Educativos, 25(99), 28-46.

Cottier, T. (2002). Trade and human rights: A relationship to discover. Journal of International Economic Law, 5, 111-132.

Cordera Campos, R., \& Santamaría Ambriz, R. (2008) Internacionalización, autonomía y calidad de la educación superior: Elementos para la integración de América Latina y el Caribe. Revista Universidades, 58(37:), 69-76.

Correia Lima, M., \& Saraiva de Alburqueque Maranhao, C. (2006) O sistema de educacao superior mundial: entre la internacionalización activa y la pasiva. Revista Avaliacao sorocaba SP, 4, 580-610.

Didou Aupetit, S. (2005) ¿Medir para conocer o para regular?: evaluación y acreditación de la educación superior en México. Avance y Perspectiva, 24(1), 9-17.

García Solá, M. (2004). El financiamiento de las instituciones de educación superior en Argentina. Caracas: IESALC/Unesco.

González, J. H. (2007). Internacionalización de la educación superior. Universidades, 33, 3-10.

Guaglianone, A. L. (2011). La conformación del sistema de educación superior en Argentina. Revista de la Educación Superior, 40(3), 164-184.

Kauffman, R., \& Haggard, S. (Comps.). (1992). The politics of economic adjustment, Princeton, New Jersey: University Press.

Larrea, M., \& Astur, A. (2014). Política internacional de educación superior. Acciones del Programa de internacionalización de la educación superior y cooperación internacional (PIESCI) 2003-2012. En E. Rinessi (Comp.), Ahora es cuando: internacionalización e integración regional universitaria en América Latina (pp. 83-98). Los polvorines: UNGS.

Marquis, C. (1998). Reflexiones sobre los cambios en las universidades argentinas. Sociológica, 13, 293-306.

Miranda, X. (2008). Integración regional e internacionalización de la educación superior en América latina y el caribe. En A. Gazzola \& A. 
Diddrikson (Eds.), Tendencias de la educación superior en América Latina y el Caribe (pp. 179-240). Caracas: IESALC/Unesco.

Mollis, M. (2007). Globalización y nuevo cosmopolitismo: la cultura empresarial en la educación superior en La educación superior en Argentina: Balance de una década. Revista de la Educación Superior, 35(2), 78-84.

Nelson, J. (Comp.). (1994). Crisis económica y políticas de ajuste. Bogotá: Editorial Norma.

Noriega, J.-E., \& Montiel, M. C. (2014). La universidad argentina entre sus regulaciones y tendencias. Revista Iberoamericana de Educación Superior, 5, 88-103.

Sebastián, J. (2011). Dimensiones y métrica de la internacionalización de las universidades. Universidades, 61, 3-16.

Solanas, F. (2011). La ley de educación superior en Argentina y la institucionalizacion de la acreditacion universitaria: de los cuestionamientos a las dificultades del cambio. Universidades, 61, 29-42.

Tobón Restrepo, L. J., Gallego, G. A., \& Yepes, V. M. (2013). Internacionalización e integración regional universitaria en América Latina educativa: del lugar común al lugar en común. Revista Virtual Universidad Católica del Norte, 40, 1-3.

Torre, J. C. (1998) Elproceso político de las reformas económicas en América Latina. Buenos Aires, Editorial Paidós.

Verger, A. (2010). WTO/GATS and the global politics of higher education. Nueva York: Routledge.

de Wit, Hans. (2011). Globalización e internacionalización de la educación superior. Universities and Knowledge Society Journal, 8, 77-84. 